\title{
Multi-objective Evolutionary Feature Selection for Fuzzy Classification
}

\author{
F. Jiménez, C. Martínez, E. Marzano, J. Palma (Member IEEE), G. Sánchez, G. Sciavicco
}

\begin{abstract}
The interpretability of classification systems refers to the ability of these to express their behaviour in a way that is easily understandable by a user. Interpretable classification models allow for external validation by an expert and, in certain disciplines such as medicine or business, providing information about decision making is essential for ethical and human reasons. Fuzzy rule-based classification systems are consolidated powerful classification tools based on fuzzy logic and designed to produce interpretable models; however, in presence of a large number of attributes, even rule-based models tend to be too complex to be easily interpreted. In this work, we propose a novel multivariate feature selection method in which both search strategy and classifier are based on multi-objective evolutionary computation. We designed a set of experiments to establish an acceptable setting with respect to the number of evaluations required by the search strategy and by the classifier, and we tested our strategy on a real-life dataset. Then, we compared our results against a wide range of feature selection methods that includes filter, wrapper, multivariate and univariate methods, with deterministic and probabilistic search strategies, and with evaluators of diverse nature. Finally, the fuzzy rule-based classification model obtained with the proposed method has been evaluated with standard performance metrics and compared with other wellknown fuzzy rule-based classifiers. We have used two real-life datasets extracted from a contact center; in one case, with the proposed method we obtained an accuracy of 0.7857 with 8 rules, while the best fuzzy classifier compared obtained 0.7679 with 8 rules, and in the second case, we obtained an accuracy of 0.7403 with 5 rules, while the best fuzzy classifier compared obtained 0.6364 with 4 rules.
\end{abstract}

Index Terms-Data Classification, Multi-objective Evolutionary Algorithms, Feature Selection, Fuzzy Rules Based Learning.

\section{INTRODUCTION}

The interpretability of classification systems refers to the ability of these to express their behaviour in a way that is easily understandable by a user. Interpretable classification models allow for external validation by an expert and, in certain disciplines such as medicine or business providing information about decision making is essential for ethical and human reasons. The fuzzy rule-based classification systems (FRBCS) [1], [2], [3], [4] have been strongly developed in the last years, and they are now consolidated powerful classification tools that also allow the interpretation of the model in a direct and clear way since they use linguistic

F. Jiménez, G. Sánchez, and J. Palma are with the Department of Information Engineering and Communications, University of Murcia, Spain, fernan,gracia,jtpalma@um.es

C. Martínez is with the International Doctorate School of the University of Murcia, Spain, carlos.martinez6@um.es

E. Marzano is CEO of G.A.P. Srl, Udine, Italy, e.marzano@gapitalia.it

G. Sciavicco is with the Department of Mathematics and Computer Science, University of Ferrara, Italy, guido.sciavicco@unife.it labels in a similar way as human reasoning does. Multiobjective Evolutionary Algorithms (MOEAs) [5] have been applied successfully in recent years for the optimization of FRBCS. There are two main motivations behind using MOEAs for FRBCS. On the one hand, evolutionary computation is a very powerful technique for the approximation of optimization and search problems in high complexity non-linear spaces, and, on the other hand, multi-objective optimization allows to simultaneously optimize the accuracy and the complexity of the FRBCS, by identifying a set of Pareto solutions. In [6], a MOEA is used to concurrently learn rule base and data base of a FRBS. In this case, two objectives are considered: the first measures the complexity as the sum of the input variable labels used in each of the rules, and the second corresponds to the mean squared error. In [7], fuzzy classifiers for imbalanced and cost-sensitive datasets are generated with a three objective MOEA. The first and second objectives are sensitivity and specificity. The third objective is a complexity measure computed as the sum of the conditions that compose the antecedents of the rules, which is minimized. In [8], PAES$R C S$ method is used to maximize accuracy and minimize the total rule length for internet traffic classification. In [9], IT2PAES-RCS extends PAES-RCS to employ Type-2 fuzzy sets, where sensitivity, specificity and total rule length are optimized for financial data classification.

However, the use of fuzzy logic may not be enough for the classification model to be interpretable. Interpretability not only implies transparency (expressibility capabilities) but also compactness. In a FRBCS, improving the compactness implies reducing the number of attributes, the number of linguistic labels for each variable, and the number of rules. Fuzzy rule-based classification systems are designed to produce interpretable models; however, in presence of a large number of attributes, the resulting classifiers may be too complex to be easily interpreted (for example, rules with more than five attributes may be intractable for a human being). In this sense, a feature selection process [10], prior to the fuzzy rule extraction phase, may be crucial step. Although feature selection problem is NP-hard [11], with a search space ranging $O\left(2^{N}\right)$ elements, where $N$ is the number of features, a heuristic or meta-heuristic search strategy can obtain good approximate solutions in reasonable times, thus reducing the complexity to build the final classifier. The three most common feature selection schemata are the so-called filter, wrapper and embedded. Filter selection methods [12], [13], [14] apply statistical measures to evaluate the attribute subset, whereas wrapper methods [15], [16] interact with a classifier to evaluate the attribute subset using some performance metric. Filter methods are computationally faster, but less accurate, than 
wrapper methods. In addition, a disadvantage of wrapper methods is that the performances of the selected subsets are often very dependent on the learning algorithm that is used for subset evaluation, so that, for example, a good selection of attributes performed with a decision tree-based wrapper method may result in a poor one when the selected attributes are used in a support vector machine. In [17] a filter feature selection method is proposed where a greedy algorithm is used as search strategy and a dependency measure between fuzzy decision and condition attributes is employed to evaluate the significance of a candidate feature. In [18] a wrapper feature selection method is proposed where best-first is used as search strategy, and Wang and Mendel method to generate fuzzy rule base is used as evaluator. Finally, embedded methods [19] achieve model fitting and feature selection simultaneously. The use of MOEAs as a search strategy for feature selection methods [20], [21], [22], [23], [24] is justified, on the one hand, by the very high cardinality of the search space, and on the other hand, by the intrinsic multi-objective nature of the problem, that requires minimizing the number of chosen attributes and maximizing some performance metrics, such as correlation, consistency, information gain, entropy, accuracy of the classifier, etc.

In [25] we proposed a FRBCS based on multi-objective evolutionary constrained real-parameter optimization, which maximizes the accuracy and minimize the number of rules, and imposes a constraint for the similarity of fuzzy sets. The maximum number of rules of the model, the maximum number of linguistic labels for each variable, and the maximum similarity of fuzzy sets, are parametrizable, so that they can be established by a user in order to obtain compact models. Once the fuzzy rule set has been extracted, a final linguistic labelling process assigns a linguistic label to each fuzzy set. In addition, this fuzzy classification method is itself a feature selection method, since it detects 'don't care conditions' attributes that can be eliminated from the classification model. However, although the classification method detects 'do not care conditions' attributes, this may not be enough in the presence of many attributes in the database, and a feature selection method prior to the fuzzy rule extraction phase is highly recommended. Therefore, if feature selection is used for a later classification based on fuzzy rules, the best choice would be a feature selection wrapper method that uses a fuzzy rulebased classifier for evaluation. However, this configuration for a wrapper method is not without drawbacks. Due to the high computational cost required by fuzzy rule-based systems in the presence of a large number of attributes, a wrapper method that uses a fuzzy rule-based classifier may be non-viable. It is therefore necessary to carefully analyse the parameters of both search strategy and evaluator to obtain a good trade-off between accuracy and run time.

In this work we propose a novel wrapper based multivariate feature selection method for FRBCS which presents the following novelties and benefits with respect to existing methods in the literature:

1) To the best of our knowledge, this is the first work for fuzzy classification that proposes a wrapper feature selection method prior to the phase of fuzzy rule extraction, where both search strategy and evaluator are performed with independent multi-objective evolutionary algorithms. In the current literature, usually, feature selection is embedded in the fuzzy rules extraction algorithm itself by identifying “don't care conditions" (embedded feature selection methods [7], [8], [9]).

2) Our method for fuzzy rule extraction consists of multiobjective constrained optimization of real parameters, instead of multi-objective combinatorial optimization as does the rest of the methods in the literature. In the rest of the methods, the rule base is explicitly separated from the (previously built) knowledge base containing the definition of the fuzzy sets. Then, the fuzzy sets are combined in the rule base using combinatorial optimization techniques [6], [7], [8], [9]. These approaches use a mixed optimization model: combinatorial optimization for rule and fuzzy sets selection, and real parameter optimization for parameter tuning. All these evolutionary algorithms use a fixed representation with triangular fuzzy sets in a Pittsburgh approach, and they use a mark equal to 0 if the rule is not selected, and a mark equal to 1 when the rule is selected, and an integer number to identify de fuzzy set, including in some cases "don't care conditions". Our approach does not build a explicit knowledge base with the definitions of the fuzzy sets, but fuzzy sets are directly embedded in the rule base in a random fashion within the domains of each variable, with a variablelength float-point representation with gaussian fuzzy sets in a Pittsburgh approach. Since the definitions of the fuzzy sets are random, this can produce intermingled and, therefore, non-interpretable partitions. To prevent this, a similarity constraint for fuzzy sets is imposed in the optimization model, which is handled by the multiobjective evolutionary algorithm by a repair technique and applied after the initialization, crossing and mutation. The gaussian fuzzy sets are represented by their centre and variance as real-coded parameters, and therefore the crossover and mutation operators used by the evolutionary algorithm are those of the float-point representations, varying the centres and the variances of the gaussian fuzzy sets separately.

3) The interpretability of the rule base can be adjusted, not only by imposing a maximum of rules and a maximum of linguistic labels, but also a maximum threshold of similarity (defined by the user) between the fuzzy sets. Thus, when the similarity threshold is close to 0 , the sets fuzzy sets are sufficiently separated, giving rise to descriptive fuzzy models, whereas when the similarity threshold is close to 1 , the fuzzy sets can be very similar, producing fuzzy approximative models (non-interpretable).

4) Our method allows to deal with databases composed of numeric as well as categorical (or nominal) attributes. This is important because in many real-life problems both types of attributes are present. Our evolutionary multi-objective algorithm treats both types of attributes separately in the representation of individuals as well as in crossing and mutation operators. Chromosomes are divided into two parts (one for the numerical attributes 
and another for the categorical ones); the numeric attributes are treated as fuzzy sets and optimized by realparameter constrained optimization, and the categorical attributes are represented with integers and optimized by combinatorial optimization. Both types of attributes are merged by the inference engine to provide the classifier's predictions.

5) Both the multi-objective evolutionary algorithm for the search strategy and for fuzzy rules extraction have been designed with self-adaptive variation operators. In this way, it is not necessary to do preliminary experimentations to adjust the crossover and mutation probabilities.

6) The search strategy and the fuzzy classifier have both been included in the Weka platform [26] as official packages. Weka is a collection of machine learning algorithms for data mining tasks. The algorithms can be applied directly to a dataset or called from proprietary Java code. Weka contains tools for data pre-processing, classification, regression, clustering, association rules, and visualization. It is also well-suited for developing new machine learning schemes. Weka is open source software issued under the GNU General Public License. The proposed feature selection method can be easily configured using the Weka Graphical User Interface. Therefore, the proposed method can be compared with the rest of feature selection methods and classifiers implemented in the platform, as well as undergo statistical tests on a wide set of performance measures.

With respect to the methodology used for the validation of the proposed method, the following techniques have been applied:

1) Due to the high computational cost, the experiments have been oriented to establishing an acceptable setting with respect to the number of evaluations required by the search strategy and by the fuzzy rule-based classifier. To this end, statistical tests have been carried out on the run time, accuracy and number of selected attributes performance metrics.

2) We compared the proposed method in its best setting to a wide range of feature selection methods (79 in total) that includes filter, wrapper, univariate, and multivariate methods, with deterministic and probabilistic search strategies, and with evaluators of diverse natures.

3) To compare the performance of the 79 feature selection methods, the accuracy, weighted area under the $R O C$ curve, root mean squared error and model size metrics have been used. To select the best methods, we propose a multi-objective decision making process to identify the non-dominated solutions of a multi-objective combinatorial optimization problem with 4 objectives (one for each performance metric).

4) Finally, the fuzzy rule-based classification model obtained with the proposed method has been evaluated with standard performance metrics and compared with other wellknown fuzzy rule-based classifiers.

For the realization of the experiments we used a real-life dataset. Operational and service data have been extracted from a medium-sized contact center that operates both inbound and outbound communications, with different purposes, including customer care and follow-up, as well as marketing and quality control, and the aim of this classification problem is to evaluate agents' performances. Operational data include all the technical information needed to reconstruct a detailed history of the events that take place during each communication, and include, for example, the dialled or dialling phone number, the agent(s) that has (have) been involved, possible call transfers, and time-stamps. On the other hand, service data are specific to the particular service for which the contact has taken place, and may include, for example, all answers given by the interviewed subject during an outbound survey. Therefore, we may define this problem as a feature selection problem, whose purpose is to establish which subset of variables best objectively indicates of the performances of an agent. To this end, the center has collected the cumulative data, represented by agent, of a significant period of time and a significant range of different services, and asked to three, independent, supervisors to evaluate each involved agent. Such an evaluation plays the role of the expert's view of this problem, and the model we are searching for tries to predict such judgement.

The paper is organized as follows. Section II introduces basic concepts of Multi-objective Constrained Optimization and two Multi-objective Evolutionary Algorithms widely used in literature. It also briefly describes the feature selection process and how feature selection methods are implemented in Weka, as well as the MultiObjectiveEvolutionarySearch and MultiObjectiveEvolutionaryFuzzyClassifier classes implemented by the authors of this work and available in the Weka platform; Section III describes the datasets of agents of a contact center used for the realization of experiments, the preprocessing phase, and the feature selection method proposed in this paper; Section IV shows the experiments performed, the parameter setting, their results, an analysis of these based on the statistical comparison with other feature selection methods and other fuzzy rule-based classifiers, and an interpretation of the fuzzy model obtained with the proposed methodology in the context of the problem. Finally, in Section V we draw some final conclusions.

\section{BACKGROUND}

Multi-objective constrained optimization. The term optimization [27] refers to selection of a best element, with regard to some criteria, from a set of alternative elements. Mathematical programming [28] deals with the theory, algorithms, methods and techniques to represent and solve optimization problems. In this paper we are interested in a class of mathematical programming problems called multiobjective constrained optimization problems [29], which can be formally defined, for $l$ objectives and $m$ constraints, as follows:

$$
\begin{array}{lll}
\text { Min./Max. } & f_{i}(\mathbf{x}), & i=1, \ldots, l \\
\text { subject to } & g_{j}(\mathbf{x}) \leq 0, & j=1, \ldots, m
\end{array}
$$

where $f_{i}(\mathbf{x})$ (usually called objectives) and $g_{j}(\mathbf{x})$ are linear or non-linear arbitrary functions. Optimization problems can be naturally separated into two categories: those with 
discrete variables, which we call combinatorial, an those with continuous variables. In combinatorial problems, we are looking for objects from a finite, or countably infinite, set $\mathcal{X}$, typically integers, sets, permutations, or graphs. In problems with continuous variables, instead, we look for real parameters belonging to some continuous domain. In (1), $\mathbf{x}=\left\{x_{1}, x_{2}, \ldots, x_{n}\right\} \in \mathcal{X}^{n}$ represents the set of decision variables, where $\mathcal{X}$ is the domain for each variable $x_{k}$, $k=1, \ldots, n$. Note that maximization problems can be defined symmetrically, and solved in an equivalent way.

Now, let $\mathcal{F}=\left\{\mathbf{x} \in \mathcal{X}^{n} \mid g_{j}(\mathbf{x}) \leq 0, j=1, \ldots, m\right\}$ be the set of all feasible solutions to (1). We want to find a subset of solutions $\mathcal{S} \subseteq \mathcal{F}$ called non-dominated set (or Pareto optimal set). A solution $\mathbf{x} \in \mathcal{F}$ is non-dominated if there is not other solution $\mathbf{x}^{\prime} \in \mathcal{F}$ that dominates $\mathbf{x}$, and a solution $\mathbf{x}^{\prime}$ dominates $\mathbf{x}$ if and only if (1) there exists $i(1 \leq i \leq l)$ such that $f_{i}\left(\mathbf{x}^{\prime}\right)$ improves $f_{i}(\mathbf{x})$, and (2) for every $i(1 \leq i \leq l), f_{i}(\mathbf{x})$ does not improve $f_{i}\left(\mathbf{x}^{\prime}\right)$. In other words, $\mathbf{x}^{\prime}$ dominates $\mathbf{x}$ if and only if $\mathbf{x}^{\prime}$ is better than $\mathbf{x}$ for at least one objective, and not worse than $\mathbf{x}$ for any other objective. The set $\mathcal{S}$ of non dominated solutions of (1) can be formally defined as:

$$
\mathcal{S}=\left\{\mathbf{x} \in \mathcal{F} \mid \nexists \mathbf{x}^{\prime}\left(\mathbf{x}^{\prime} \in \mathcal{F} \wedge \mathcal{D}\left(\mathbf{x}^{\prime}, \mathbf{x}\right)\right)\right\}
$$

where:

$$
\begin{aligned}
\mathcal{D}\left(\mathbf{x}^{\prime}, \mathbf{x}\right) \equiv & \exists i\left(1 \leq i \leq l, f_{i}\left(\mathbf{x}^{\prime}\right)<f_{i}(\mathbf{x})\right) \wedge \\
& \forall i\left(1 \leq i \leq l, f_{i}\left(\mathbf{x}^{\prime}\right) \leq f_{i}(\mathbf{x})\right) .
\end{aligned}
$$

Once the set of optimal solutions is available, the most satisfactory one can be chosen by applying a preference criterion. When all the functions $f_{i}$ are linear, then the problem is a linear programming problem [30], which is the classical problem of mathematical programming and extremely efficient algorithms exist to obtain the optimal solution (e.g., the simplex method [31]). When any of the functions $f_{i}$ is nonlinear then we have a non-linear programming problem [32]. A non-linear programming problem in which the objectives are arbitrary functions is, in general, intractable. In principle, any search algorithm can be used to solve combinatorial optimization problems, although they are not guaranteed to find an optimal solution. Metaheuristics methods such as evolutionary algorithms [33] are typically used to find approximate solutions for complex multi-objective optimization problems, including feature selection and fuzzy classification.

The multi-objective evolutionary algorithms ENORA and NSGA-II. The multi-objective evolutionary algorithms ENORA [25], [34], [35], [36], [37] and NSGA-II [5], [38] use a $(\mu+\lambda)$ strategy with $\mu=\lambda=$ popsize, where $\mu$ corresponds to the number of parents and $\lambda$ refers to the number of children (popsize is the population size), with binary tournament selection and a rank function based on Pareto fronts and crowding. The difference between NSGA-II and ENORA is how the calculation of the ranking of the individuals in the population is performed. In ENORA each individual belongs to a slot (as established in [37]) of the objective search space, and the rank of an individual in a population is the non-domination level of the individual in its slot. In NSGA-II, the rank of an individual in a population is the non-domination level of the individual in the whole population. ENORA uses the fast nondominated sorting as NSGA-II. It compares each solution with the rest of the solutions and stores the results to avoid duplicate comparisons between every pair of solutions. For a problem with $l$ objectives and a population with $P$ solutions, the fast non-dominated sorting needs to conduct $l \cdot P \cdot(P-1)$ objective comparisons, which means that it has a algorithmic complexity of $O\left(l \cdot P^{2}\right)$. However, ENORA distributes the population in $P$ slots (in the best case), therefore, the algorithmic complexity of ENORA is $O\left(l \cdot P^{2}\right)$ in the worst case, and $O(l \cdot P)$ in the best case.

Feature selection. Feature Selection is defined in [10] as the process of eliminating features from the dataset that are irrelevant to the task to be performed. It facilitates data understanding, reduces the measurement and storage requirements, reduces the computation time, and reduces the size of a dataset, so that model learning becomes an easier process. A selection method is basically a search strategy where the performance of candidate subsets is measured with a given evaluator. The search space for candidate subsets has cardinality $O\left(2^{n}\right)$, where $n$ is the number of features. A stopping criterion establishes when the feature selection process must finish. It can be defined as a control procedure that ensures that no further addition or deletion of features does produce a better subset, or it can be as simple as a counter of iterations.

As discussed in the Introduction section, feature selection methods are typically categorized into wrapper, filter and embedded, as well as, orthogonally, into univariate and multivariate methods. Wrapper methods [15] use a predetermined learning algorithm to determine the quality of selected features by using some evaluation metric [16]; filter methods apply statistical measures to evaluate the set of attributes [12], [13], [14], while embedded methods achieve model fitting and feature selection simultaneously [19]. Finally, multivariate methods evaluate features in batches, while univariate methods rank each feature independently of the feature space.

Multi-objective evolutionary search. We use in this paper our MultiObjectiveEvolutionarySearch package of Weka. Our search strategy identifies non-dominated solutions to the following multi-objective optimization problem, which can be formulated as an instance of the problem (1) with $l=2$ (two objectives) and $m=0$ (no constraints) [39]:

$$
\begin{array}{ll}
\text { Max./Min. } & \mathcal{F}_{\mathcal{D}}(\mathbf{x}) \\
\text { Min. } & \mathcal{C}(\mathbf{x})
\end{array}
$$

where $\mathbf{x}=\left\{x_{1}, x_{2}, \ldots, x_{n}\right\}$ is a boolean set of decision variables, i.e. $x_{k} \in\{$ true, false $\}, k=1, \ldots, n$, being $n$ the number of input attributes of a database $\mathcal{D}$. The problem (2) is, therefore, a multi-objective boolean combinatorial optimization problem where $x_{k}=1$ represents that the variable $x_{k}$ is selected, and $x_{k}=0$ represents that the variable $x_{k}$ is not selected, for all $k=1, \ldots, n$. Depending on the type of the $\mathcal{F}_{\mathcal{D}}(\mathbf{x})$ function, which is defined with the evaluator, the resulting selection method can be a filter (when $\mathcal{F}_{\mathcal{D}}(\mathbf{x})$ is a statistical measure over the database $\mathcal{D}$ ) or a wrapper (when $\mathcal{F}_{\mathcal{D}}(\mathbf{x})$ is a performance measure - to be minimized 
or maximized according to the metric - that evaluates the performance of a learning algorithm over the database $\mathcal{D}$ ). The function $\mathcal{C}(\mathbf{x})$ measures the number of selected features, i.e.

$$
\mathcal{C}(\mathbf{x})=\sum_{k=1}^{n} \eta\left(x_{k}\right)
$$

where $\eta$ is a function that transforms a boolean value into numeric (true $=1$ and false $=0$ ).

To solve (2), ENORA and NSGA-II have been implemented with fixed-length binary representation, uniform random initialization, binary tournament selection, ranking based on nondomination level with crowding distance, and self-adaptive uniform crossover and one flip mutation operators.

Multi-objective evolutionary fuzzy classification. We use in this paper our MultiObjectiveEvolutionaryFuzzyClassifier package of Weka. Our method identifies non-dominated solutions (fuzzy rule-based classifiers) of the following multiobjective constrained optimization problem, which can be formulated as an instance of the problem (1) with $l=2$ (two objectives) and $m=4$ (four constraints) [25]:

$$
\begin{array}{ll}
\text { Max./Min. } & \mathcal{F}_{\mathcal{D}}(\boldsymbol{\Gamma}) \\
\text { Min. } & \mathcal{N} \mathcal{R}(\boldsymbol{\Gamma}) \\
\text { subject to }: & \mathcal{N} \mathcal{R}(\boldsymbol{\Gamma}) \geq w \\
& \mathcal{N} \mathcal{R}(\boldsymbol{\Gamma}) \leq M_{\max } \\
& \mathcal{N} \mathcal{L}(\boldsymbol{\Gamma}) \leq L_{\max } \\
& \mathcal{S}(\boldsymbol{\Gamma}) \leq g_{s}
\end{array}
$$

where $\Gamma$ is a fuzzy rule-based classifier composed by $\mathcal{N} \mathcal{R}(\Gamma)$ fuzzy rules. Each fuzzy rule $R_{j}^{\Gamma}, j=1, \ldots, \mathcal{N} \mathcal{R}(\boldsymbol{\Gamma})$ has the following structure:

$$
\begin{aligned}
R_{j}^{\Gamma}: & \text { if } x_{1} \text { is } A_{1 j}^{\Gamma} \wedge \ldots \wedge x_{p} \text { is } A_{p j}^{\Gamma} \wedge \\
& y_{1} \text { is } B_{1 j}^{\Gamma} \wedge \ldots \wedge y_{q} \text { is } B_{q j}^{\Gamma} \quad \rightarrow z i s C_{j}^{\Gamma},
\end{aligned}
$$

where $x_{i} \in\left[l_{i}, u_{i}\right] \subset \mathbb{R}, i=1, \ldots, p, p \geq 0$, are real input attributes, $y_{i} \in\left\{1, \ldots, v_{i}\right\}, i=1, \ldots, q, q \geq 0, v_{i}>1$, are categorical input attributes, and $z \in\{1, \ldots, w\}, w>1$ is a categorical output attribute. Each fuzzy set $A_{i j}^{\Gamma}, i=1, \ldots, p$, $j=1, \ldots, \mathcal{N} \mathcal{R}(\boldsymbol{\Gamma})$ is defined with a gaussian membership function [40]. In the problem (3), the function $\mathcal{F}_{\mathcal{D}}(\boldsymbol{\Gamma})$ is a performance measure of the classifier $\Gamma$ over the database $\mathcal{D}$. The function $\mathcal{N} \mathcal{R}(\boldsymbol{\Gamma})$ is minimized, and the constraints $\mathcal{N} \mathcal{R}(\boldsymbol{\Gamma}) \geq w$ and $\mathcal{N} \mathcal{R}(\boldsymbol{\Gamma}) \leq M_{\max }$ limit the number of rules of the classifier $\boldsymbol{\Gamma}$ to the interval $\left[w, M_{\max }\right]$, where $w$ is the number of classes of the output attribute, and $M_{\max }$ is given by user. The constraint $\mathcal{N} \mathcal{L}(\boldsymbol{\Gamma}) \leq L_{\max }$ limits the number of linguistic labels of the real input variables to $L_{\max }$. Finally, the constraint $\mathcal{S}(\boldsymbol{\Gamma}) \leq g_{s}$ ensures a maximum similarity $g_{s}$ $\left(0<g_{s} \leq 1\right)$ between the fuzzy sets; the similarity value of a classifier $\Gamma$ represents the maximum value of overlapping among their fuzzy sets for any input variable. The constraint $\mathcal{S}(\boldsymbol{\Gamma}) \leq g_{s}$ is handled by the multi-objective evolutionary algorithm by means of a repair algorithm, which is applied after the initialization of solutions, and after the crossing and mutation.
As reasoning method we use the maximum matching where, the compatibility degree of the rule $R_{j}^{\Gamma}$ for the example $(\mathbf{x}, \mathbf{y})$ is calculated as:

$$
\varphi_{j}^{\Gamma}(\mathbf{x}, \mathbf{y})=\left(\phi_{j}^{\Gamma}(\mathbf{y})+1\right) \prod_{i=1}^{p} \mu_{A_{i j}^{\Gamma}}\left(x_{i}\right)
$$

where $\phi_{j}^{\Gamma}(\mathbf{y})$ is the number of categorical input attributes, so that $y_{i}=B_{i j}^{\Gamma}$. The compatibility degree is obtained by applying a t-norm product to the degree of satisfaction of the clauses $x_{i}$ is $A_{i j}^{\Gamma}$ multiplied by the number of matches of the categorical input data $y_{i}$ is $B_{i j}^{\Gamma}$. The association degree of the example $(\mathbf{x}, \mathbf{y})$ with the class $C$, is calculated by summing the compatibility degrees of each rule $R_{j}^{\Gamma}$ whose value for the categorical output attribute $C_{j}^{\Gamma}$ is equal to $C$, that is:

$$
\lambda_{C}^{\Gamma}(\mathbf{x}, \mathbf{y})=\sum_{\substack{j=1, \ldots, M_{\Gamma} \\ C_{j}^{\Gamma}=C}} \varphi_{j}^{\Gamma}(\mathbf{x}, \mathbf{y})
$$

The classification for the example $(\mathbf{x}, \mathbf{y})$ or output of the classifier $\Gamma$, corresponds to the class $C$ whose association degree is maximum, that is:

$$
f_{\Gamma}(\mathbf{x}, \mathbf{y})=\arg _{C} \max _{C=1}^{w} \lambda_{C}^{\Gamma}(\mathbf{x}, \mathbf{y})
$$

For the problem (3), ENORA and NSGA-II have been implemented with variable-length representation with float-point and categorical input variables with a Pittsburgh approach, uniform random initialization, binary tournament selection, handling constraints using a repair algorithm, ranking based on non-domination level with crowding distance, self-adaptive variation operators which work on different levels of the fuzzy classifier: fuzzy set crossover, rule crossover, rule incremental crossover, gaussian set center mutation, gaussian set variance mutation, fuzzy set mutation, rule incremental mutation, and integer mutation (for categorical data). Once the fuzzy rule set has been extracted, a linguistic label is assigned to each fuzzy set.

\section{Assessing Agents' Quality in a Contact CENTER}

Evaluating the quality of the work that is being done by the employees is a central problem in modern business. Such an evaluation should be correct, fair, systematic and reliable, and, to this end, it should be measurable. For the purpose of experimenting the capabilities of our feature selection/fuzzy classification schema, we considered the problem of evaluating the quality of the work of operators (also called agents) in a medium-sized contact center. A call center is a set of resources, personnel, computers, and telecommunication equipment, which enable the delivery of services via telephone. Thanks to the advancements in information technology, call centers are gradually evolving into contact centers, in which the phone-operator role of agents is complemented, and sometimes substituted, by services offered through other technologies, such as faxing, instant messaging, web portals, and so on. Contact centers handle both inbound and outbound 
communications, with different purposes, including customer care and follow-up, as well as marketing and quality control. As we shall see, compared to previous data mining experiments on contact center databases, the quality of the information at our disposal is considerably higher. Not only previous experiments such as [41] made no use of feature selection; they also operated on a very restricted set of attributes, consequently limiting the significance of their results. Moreover, all previous experiments, including [35], [42], were not designed to evaluate the performances of the agents.

Datasets. The data we have used have been provided by Northern Italy contact center GAP S.R.L., and consists of the cumulative performances data of 77 agents over a period of 6 months. Contacts in GAP are managed and organized as follows. The flux of information is categorized into inbound (that is, contacts that GAP receives, such as phone calls) and outbound (i.e., surveys made by GAP). Each of these is classified by commissions: a commission is the unit of contract between GAP and a client (i.e., the ACME airline commissions to GAP the phone ticket selling service for their customers), and each commission may be declined into several services. A service is a specific type of interaction that the client wants GAP to operate with (i.e., ACME wants GAP to deal with ticket selling but not lost-and-found), and each service includes several sub-types (i.e., ACME ticket selling includes a channel for information, a channel for reservation managing, and so on). For the purpose of this experiment, we considered phone-based communications only. Of all agents, 56 were employed for outbound, inbound, and back office services, while the remaining 21 had no inbound communications, naturally leading to two datasets: $A L L \_A G E N T S$ (i.e., those who managed only outbound communications and back office services) and INBOUND_AGENTS (i.e., those who managed, among others, also inbound communications). The work of all agents has been described via 69 attributes, while for those agents with at least some inbound communications over the analysed period, we were able to add 6 more features (that make sense for inbound communications only).

The set of variables common to both datasets (the one containing the cumulative performance indicators of all agents and the one containing the cumulative performance indicators of only those agents that had inbound communications) can be classified into several categories, depending on the particular aspect they describe, for a better understanding. The first category is agent related variables (see Table I - top ${ }^{1}$ ), and includes their seniority (from 6 months to 5 and an half year), their gender ( 31 males versus 46 females), their age (from 19 to 65 years old), their level of education (from 1 - minimum compulsory education, to 5 - university degree or more), and their skill average and variance: GAP has internally engineered a skill-function that takes into account several aspects, recomputed weekly for each agent, and of which we consider the average and the variance over the entire period. A second category of variables is work's diversity, by means of which we want to measure how heterogeneous has been the agent's work

\footnotetext{
${ }^{1}$ Unless otherwise specified, every numeric variable is in fact a pair of variables that takes into account average and variance of each aspect.
}

in the analysed period. This category includes the number of distinct sessions ${ }^{2}$ and distinct commissions the agent has worked on, the daily frequency of context switches (that takes into account switching between flows, or services, or service sub-types, weighted: farthest jumps weight the most), the daily frequency of flow switches (inbound vs. outbound), the daily frequency of service switches, and the the daily frequency of sub-type switches, and it is given in Table I (bottom). Moreover, we have taken into account how the agents' work has been distributed (Table II - top), by including the average and the variance over days of the number of minutes during which he/she has been effectively working (management), on inbound (management inbound), on outbound (management outbound) communications, or on back office (management back office), along with their fraction on the entire workload, that takes into account how many times the agent has declared him/herself available (in idle state), for how many minutes in total, on break, and for how many minutes, and inactive (that is, on break or available). The distribution takes also into account the icc index, which is an internal evaluation of the importance, complexity and criticality of the service being worked on. Finally, Table II (bottom) shows the variables relative to agents' turns distribution, that take into account in which part of the day and of the week each agent's shifts are mainly scheduled, as well as the fraction, over the entire observed period, of break, available, and inactive time of the agent.

Six more attributes have been considered for those agents whose job during the observed period included inbound communications. Such variables take into account the structure, the understandability, and the type of call-related notes written by the agent. These may be articulated, non-articulated, domainrelated, hybrid, or unrecognizable.

The dataset $A L L \_A G E N T S$ contains 69 input attributes and 77 instances, while the dataset INBOUND_AGENTS contains 75 input attributes and 56 instances. Both datasets have been enriched with a variable that describes the agent performance value. This has been obtained by asking to three independent supervisors a fair judgement of each agent to the best of their expertise. Their judgement, on a scale from 1 (lowest) to 5 (highest), takes into account the overall impression of the agents and their performances. Then, the three votes have been combined into a single one by averaging them. Four class labels are identified according to the average judgement: 'Low' [1,2), 'Medium' [2,3), 'High' [3,4), 'Excellent' $[4,5]$.

Preprocessing. For each of the two datasets we applied a simple preprocessing methodology. First, we have replaced all the missing values with their respective mean; to this end, the class weka.filters.unsupervised.attribute.ReplaceMissingValues has been used. Second, we have searched for those features with too small variation: no features have been eliminated via this process, indicating that, potentially, all of them might influence the agent judgement. We have used

\footnotetext{
${ }^{2} \mathrm{~A}$ session is the most basic unit of work done by the agent, to which it is possible to assign a result, for example a phone call.
} 


\begin{tabular}{|c|c|}
\hline \multicolumn{2}{|r|}{ Agent related variables } \\
\hline attribute & semantics \\
\hline agent_seniority & \# of days of service of the agent \\
\hline agent_gender & whether the agent is a male or female \\
\hline agent_age & age of the agent \\
\hline agent_education & level of education of the agent \\
\hline agent_skill & weekly avg. and var. of agents' skill \\
\hline & Diversity variables \\
\hline attribute & semantics \\
\hline num_sessions & daily avg. and var. of the \# of distinct sessions \\
\hline num_commissions & daily avg. and var. of the \# of distinct commissions \\
\hline switch_index & daily avg. and var. of (all) switches \\
\hline switch_index_flow_type & daily avg. and var. of flow switches \\
\hline switch_index_ser_type & daily avg. and var. of service switches \\
\hline switch_index_ser_same_type & daily avg. and var. of sub-service type switches \\
\hline icc_inbound_av & daily avg. and var. of avg. icc index in inbound \\
\hline icc_outbound_av & daily avg. and var. of avg. icc index in outbound \\
\hline icc_inbound_var & daily avg. and var. of var. icc index in inbound \\
\hline icc_outbound_var & daily avg. and var. of var. icc index in outbound \\
\hline
\end{tabular}

TABLE I

VARIABLES RELATED TO THE AGENT AND VARIABLES RELATED TO THE SWITCHING FREQUENCY OF THE AGENT.

\begin{tabular}{|c|c|}
\hline \multicolumn{2}{|r|}{ Work distribution variables } \\
\hline attribute & semantics \\
\hline management & daily avg. and var. of \# min. working \\
\hline management_inbound & daily avg. and var. of \# min. working on inbound comm. \\
\hline management_outbound & daily avg. and var. of \# min. working on outbound comm. \\
\hline management_backoffice & daily avg. and var. of \# min. working on back office \\
\hline fraction_inbound & daily avg. and var. of the $\%$ of min. on inbound \\
\hline fraction_outbound & daily avg. and var. of the $\%$ of min. on outbound \\
\hline fraction_backoffice & daily avg. and var. of the $\%$ of min. on back office \\
\hline available_sessions & daily avg. and var. of the \# of available sessions \\
\hline available & daily avg. and var. of \# min. available \\
\hline break_sessions & daily avg. and var. of the \# of break sessions \\
\hline break & daily avg. and var. of \# min. on break \\
\hline inactive_sessions & daily avg. and var. of the \# of inactive sessions \\
\hline inactive & daily avg. and var. of \# min. inactive \\
\hline \multicolumn{2}{|r|}{ Turn distribution variables } \\
\hline attribute & semantics \\
\hline turn_duration & daily avg. and var. of turn length in \# min. \\
\hline fraction_weekend & fraction of weekend workdays \\
\hline fraction_night & daily avg. and var. of the $\%$ of min. working during nights \\
\hline fraction_morning & daily avg. and var. of the $\%$ of min. working during mornings \\
\hline fraction_early_afternoon & daily avg. and var. of the $\%$ of min. working during early aft. \\
\hline fraction_late_afternoon & daily avg. and var. of the $\%$ of min. working during late aft. \\
\hline fraction_evening & daily avg. and var. of the $\%$ of min. working during evening \\
\hline inactivity_time & fraction of total inactivity time over total turn duration \\
\hline available_time & fraction of total availability time over total turn duration \\
\hline break time & fraction of total break time over total turn duration \\
\hline
\end{tabular}

TABLE II

VARIABLES RELATED TO THE AGENT'S WORK DISTRIBUTION AND HETEROGENEITY, AND TURN DISTRIBUTION.

the class weka.filters.unsupervised.attribute.RemoveUseless for this task.

Feature selection for fuzzy classification. As we have mentioned, one of the drawbacks of fuzzy classification is that it generates little interpretable models in the presence of many attributes. In these cases, a process of selection of attributes, prior to fuzzy rule extraction, is required. In this paper we propose the following multivariate wrapper feature selection method for later use in fuzzy classification. As for the search strategy, we propose our MultiObjectiveEvolutionarySearch method with the multi-objective evolutionary algorithm ENORA. As shown in [35], [36], the performance of ENORA are generally better than those of NSGA-II in terms of hypervolume [43], and better than other single-objective search strategies. Our evaluator consists of a wrapper with the fuzzy rule-based classifier based, again, on ENORA, which outperforms NSGA-II in this task as well [25], driven by the

\begin{tabular}{ll}
\hline \multicolumn{2}{c}{ Notes' structure variables } \\
\hline attribute & semantics \\
\hline fraction_abbreviated & fraction of abbreviated notes \\
fraction_articulated & fraction of articulated notes \\
fraction_non_articulated & fraction of non articulated notes \\
fraction_hybrid & fraction of hybrid notes \\
fraction_unrecognized & fraction of unrecognized notes \\
fraction_domain & fraction of domain-related notes \\
\hline \multicolumn{3}{c}{ TARIABLES RELATED TO THE AGENT'S NOTES. }
\end{tabular}

accuracy $(A C C)$ as a measure. The $A C C$-guided search has given better results in the preliminary experiments than the search using the area under the ROC curve $(A U C)$, which gives very poor values of $A C C$. As stopping criterion we use a simple limit on the number of generations. The proposed method basically consists of a multi-objective evolutionary algorithm (search strategy) where, for the evaluation of a candidate attribute subset, a wrapper (WrapperSubsetEval) based on the MultiObjectiveEvolutionaryFuzzyClassifier classifier and accuracy performance metric is used. Therefore, to evaluate a candidate attribute subset, a multi-objective evolutionary algorithm is executed to extract a fuzzy rule set on the reduced database, which is evaluated with cross-validation on the accuracy metric. The following steps are then performed for the evaluation of an attribute subset $\mathbf{x}=\left\{x_{1}, x_{2}, \ldots, x_{N}\right\}$, $x_{k} \in\{$ true false $\}, k=1, \ldots, N$, in a database $\mathcal{D}$ :

STEP 1. Remove from the database $\mathcal{D}$ those attributes $x_{k}$ such that $x_{k}=$ false, obtaining a reduced database $\mathcal{D}$ ';

STEP 2. Run MultiObjectiveEvolutionaryFuzzyClassifier over the database $\mathcal{D}$ ' to extract a fuzzy rule set.

STEP 3. Evaluate the fuzzy rule set with cross-validation using the accuracy performance metric;

STEP 4. Return the 'merit' of the attribute subset $\mathbf{x}$.

Considering the wideness of the search space $\left(O\left(2^{N}\right)\right)$ for feature selection, and the intrinsic complexity of fuzzy rule extraction (for each attempted candidate), it is crucial to adjust the evolution parameters of both evolutionary multi-objective algorithms to obtain a good trade-off between accuracy and run time.

\section{EXPERIMENTS, RESULTS AND DISCUSSION}

In this section, we describe the set of experiments that have been performed to show the effectiveness of the proposed methodology. Three blocks of experiments have been carried out: the first one aims to find the optimal number of generations of both the search strategy and the evaluator for an adequate trade-off between performance and run time; in the second block, the proposed method is compared with other multivariate, univariate, filter and wrapper feature selection methods; in the third block, we compare the fuzzy classifiers obtained by the proposed method with the fuzzy classifiers obtained by well-known methods. Finally, the rules of our best fuzzy models are interpreted in the context of the contact centre. All our experiments have been run on a machine with 8 processors Intel Xeon X7550 at $2.00 \mathrm{GHz}, 1 \mathrm{TByte}$ of RAM at $1067 \mathrm{MHz}$, and storage Lustre Distributed File System v2.5.2 - Interconnection network: Infiniband QDR (40Gbps). 


\begin{tabular}{ccc}
\hline $\begin{array}{c}\# \\
\text { Configuration }\end{array}$ & $\begin{array}{c}\text { Number of generations } \\
\text { Evolutionary search }\end{array}$ & $\begin{array}{c}\text { Number of generations } \\
\text { Fuzzy classifier }\end{array}$ \\
\hline$\# 1$ & 10 & 10 \\
$\# 2$ & 10 & 100 \\
$\# 3$ & 100 & 10 \\
\hline
\end{tabular}

TABLE IV

THREE PARAMETER CONFIGURATIONS STUDIED IN THIS PAPER.

\begin{tabular}{cccc}
\hline Configuration & Run time & Accuracy & Number of attributes \\
\hline \multicolumn{4}{c}{ INBOUND_AGENTS } \\
\hline$\# 1$ & 158300955.5 & 0.50607 & 9.7 \\
$\# 2$ & 1140188340.7 & 0.57998 & 6.0 \\
$\# 3$ & 553075217.9 & 0.56 & 3.5 \\
\hline \multicolumn{4}{c}{ ALL_AGENTS } \\
$\# 1$ & 148756602.7 & 0.50857 & 7.6 \\
$\# 2$ & 1200222302.3 & 0.56128 & 8.0 \\
$\# 3$ & 688512492.7 & 0.54831 & 5.5 \\
\hline
\end{tabular}

TABLE V

AVERAGE RUN TIME, ACCURACY AND NUMBER OF ATTRIBUTES

Optimal number of generations. Given that the proposed feature selection method consists of an evolutionary algorithm that, in turn, uses another evolutionary algorithm to evaluate each individual population, the run time may be intolerable if the number of generations for each of the evolutionary algorithms is not properly adjusted. To this aim, we have established three different configurations, shown in the Tab. IV. We executed 10 times our feature selection method in each of the three configurations. Table $\mathrm{V}$ shows a summary of results with the averages of run time (ms.), accuracy and number of selected attributes. In order to test if the differences between the means of each of the configurations are statistically significant, statistical tests have been performed. First, the conditions to apply the parametric test one way repeated measures ANOVA, normality and sphericity, have been checked. Only the dataset $A L L \_A G E N T S$ for the number of attributes meets these conditions (Shapiro-Wilk normality test p-values $0.119,0.381$ and 0.8 for configurations \#1, \#2 and \#3 respectively; Mauchly test for sphericity p-value 0.245 ). In this case, one way repeated measures ANOVA has been performed. For the rest of cases, Friedman test has been applied. When statistically significant differences are detected in the Friedman test, the Nemenyi post-hoc test has been applied to locate where these differences are.

Regarding the run time, configuration \#1 was the best one, as expected. Configuration \#3 behaved better than the configuration \#2, although the differences are not statistically significant. Regarding accuracy, no statistically significant differences between the configurations \#2 and \#3 were found, and both behaved better than configuration \#1. Finally, regarding the number of selected attributes, there are no statistically significant differences between the three configurations. This analysis allowed us to conclude that the worst configuration is \#1 regarding to accuracy and, since there are no statistically significant differences between configurations \#2 and $\# 3$, we opted for configuration \#3 according to the principle of minimum description length. Therefore, configuration \#3 has been used for feature selection. The remaining parameters were set as follows: populationSize was 100 in both the search

\begin{tabular}{llcr}
\hline Dataset & Selected attributes & Rank & Importance \\
\hline INBOUND_AGENTS & switch_index_ser_type & 3 & 0.01071 \\
& switch_index_ser_same_type & 4 & -0.00357 \\
& icc_inbound_av & 2 & 0.02857 \\
& turn_duration & 1 & 0.06786 \\
\hline ALL_AGENTS & management & 1 & 0.418 \\
& break_sessions & 4 & 0.301 \\
& icc_inbound_var & 3 & 0.348 \\
& fraction_night & 2 & 0.377 \\
\hline
\end{tabular}

TABLE VI

SELECTED ATTRIBUTES AND THEIR RANKS WITH THE PROPOSED FEATURE SELECTION METHOD FOR INBOUND_AGENTS AND ALL_AGENTS DATASETS.

and the classification; maxRules was 14 (i.e., 10 plus the number of classes); maxSimilarity was 0.1 (allowing only minimally overlapping fuzzy sets); $\min V$ was 2.0 and $\max V$ was 30.0 (default values). Among the 3000 individuals of the final population in the model with configuration \#3, we have chosen the one with the best accuracy; given that the number of chosen attributes is low enough, no further a posteriori decision method was necessary.

Identifying the best attributes and their importance. Tab. VI shows the selected attributes and their ranks and importances for each of the datasets. The rank and importance of the attributes has been obtained through a univariate wrapper feature selection method, where the search strategy is the ranker method, and the evaluator is ClassifierAttributeEval with classifier $=$ MultiObjectiveEvolutionaryFuzzyClassifier (algorithm $=$ ENORA, generations $=1000$, populationSize $=$ 100 , evaluationMeasure $=A C C$, maxLabels $=3$, maxRules $=4$, maxSimilarity $=0.4, \min V=30.0, \max V=2.0$, seed $=1$, , evaluationMeasure $=A C C$, and leaveOneAttributeOut = true. An attribute is evaluated by measuring the impact of leaving it out from the full set. Before analyzing the behaviour of our method from the numerical point of view, and comparing its performances with those of other methods, let us observe how the selected features may help assessing the quality of agents' work. Recall that each agents has been previously classified by three, independent experts, and that the combination of their judgment has been used as class. As for the dataset INBOUND_AGENTS, the most important attribute that has been selected is turn_duration, while the second most important is icc_inbound_av. This means that in order to automatically predict the quality of an agent with inbound communications, the average duration of their turn, and the average complexity of their task are key values. On the other hand, for agents with outbound tasks only (ALL_AGENTS dataset), the average quantity of -effective- working time, and how much of the agents' work takes place during night, seem to determine his/her quality. These elements can be used to design a simple system that, using the rules discussed later, may help the management to optimize turn and work distribution to ensure higher standards.

Comparing the results with other selection methods. The experiments performed in this section aim to answer the following questions:

1) Which feature selection method produces the best performance for fuzzy classification? 
2) How does the proposed feature selection method behave with other classifiers?

In order to answer these questions we have systematically applied a very wide range of feature selection methods, all available in the literature. Each method is, in itself, a combination of a specific choice among the search strategies, the evaluators, and the evaluation metrics (in the case of wrapper methods). In the following, we describe our choices for search methods and evaluators.

Let us consider possible search methods. As for univariate methods, we used the Ranker method [44], which ranks attributes by their individual evaluations. As for multivariate methods, among deterministic search strategies we considered: BestFirst [45], GreedyStepwise [46], LinearForwardSelection [47], and InfoGain [48], while the employed probabilistic algorithms are: MultiObjectiveEvolutionarySearch (already described in Section II), PSOSearch [49], and GeneticSearch [50]. BestFirst implements beam search, and searches the space of attribute subsets by greedy hill climbing augmented with a backtracking capability; the amount of backtracking may be customized by specifying the beam width. It supports forward, backward, and bi-directional search directions. GreedyStepwise performs a greedy forward or backward search through the space of attribute subsets, stopping when the addition (forward direction) or deletion (backward direction) of any of the remaining attributes results in a decrease in evaluation, thus, it has no backtracking capability. LinearForwardSelection is an extension of BestFirst, supporting simple forward or floating forward search directions. The latter considers a number of consecutive single-attribute elimination steps after each forward step, as long as this results in an improvement. The algorithm takes only a restricted number of $k$ attributes into account, with the goal of reducing the number of evaluations performed during the search and producing a compact final subset, by two possible modes of operation: fixed-set or fixed-width. According to the former, all single attributes are initially ranked, and then the top- $k$ are passed as input to forward selection. The latter employs a similar initial ranking criterion, starting the search with the top- $k$ attributes; however, it maintains a fixed number of $k$ candidates also in each of the subsequent forward selection steps, by adding further attributes from the initial ranked list (as long as any remain). Finally, the InfoGain strategy works by listing all features, ordered by their individual scores, as determined by measuring the information gain score with respect to the class. As far as probabilistic algorithms are concerned, genetic (or evolutionary) algorithms are the most common choice. Genetic algorithms were first proposed for attribute selection in [51], and are now considered an important tool for the selection of features [52]. They are inspired by the process of natural selection and, through the application of elitist selection, iteratively generate better and better solutions to optimization and search problems, by employing operators such as mutation and crossover. The goodness of a solution is determined through the use of one (single-objective) or more (multi-objective) fitness functions. In the present work, for the purpose of attribute selection (in those cases in which we choose multi-objective optimization), two objectives are optimized: the first one is chosen by the evaluator, and it is to be maximized, while the second one is the attribute subset cardinality, and it is to be minimized. The final output is given by the non-dominated solution in the last population having the best fitness score for the first objective. GeneticSearch implements the simple, classical Goldberg's (single-objective) Genetic Algorithm for searching. Finally, PSOSearch explores the attribute space employing the Particle Swarm Optimization (PSO) algorithm. PSO optimizes a problem iteratively, trying to improve a candidate solution with regard to a given measure of quality. Similarly to evolutionary computation techniques, it considers a population of candidate solutions, called particles. Elements are moved around the search space according to mathematical formulae, considering each particle's characteristics and the overall "swarm knowledge", following an agentoriented paradigm.

Now, let us examine the different evaluators that we considered. As much as multivariate filters are concerned, we used CfsSubsetEval [53] and ConsistencySubsetEval [54]. CfsSubsetEval evaluates the worthiness of an entire subset of features by considering the individual predictive power of each attribute, together with the degree of redundancy between them. Subsets containing attributes that are highly correlated with the class, and not strongly correlated with one another, are preferred. On the contrary, ConsistencySubsetEval scores a subset of features as a whole, by projecting the training instances according to the attribute subset, and considering the consistency of class values in the obtained instance sets. For possible univariate filters, GainRatioAttributeEval [55], SignificanceAttributeEval [56] and SymmetricalUncertAttributeEval [57] were considered. GainRatioAttributeEval evaluates the worthiness of a single attribute by measuring its gain ratio value with respect to the class labels. Gain ratio is a well-known, commonly used assessment measure, calculated as the difference between the entropy of class distribution minus the conditional entropy of the classes given the values of the attribute, divided by the entropy of the attribute itself. SignificanceAttributeEval scores a single attribute by computing its probabilistic significance as a two-way function of its association to the class decision. The intuition behind this algorithm is that if an attribute is significant with respect to the class labels, then it is expected that different sets of elements with complementary sets of values for the attribute will also belong to complementary sets of classes. Finally, SymmetricalUncertAttributeEval evaluates the worthiness of a given attribute by measuring its symmetrical uncertainty with respect to the class. Finally, as possible wrappers, we used WrapperSubsetEval [15] for multivariate methods and ClassifierAttributeEval [58] for univariate methods, in conjunction with the classifiers J48 (C4.5 [19]), LibSVM [59] and RandomForest [60], and with the metrics ACC, weighted area under the ROC curve (WAUC), and root mean squared error (RMSE). J48 is a Java implementation of the widely-used decision tree learner $C 4.5$, which is known to be computationally efficient. The learning algorithm builds a decision tree from a set of labelled training instances in a recursive fashion, starting from the root node, by using the information gain 
ratio criterion. LibSVM is a library for support vector machines learning. A support vector machine is a supervised machine learning algorithm, which can be used for both regression and (typically binary) classification problems. Each instance is mapped to a point in $n$-dimensional space, where $n$ is the number of features characterizing the instance. Then, in a binary classification setting, a hyperplane is constructed, that optimally divides the instances in homogeneous groups with respect to the class labels. RandomForest is an ensemble learning method which constructs a forest of random trees, for classification or regression purposes. A typical problem of decision trees is their propensity to overfit, if not properly pruned: in the literature, they are regarded as models having low bias, but high variance. In RandomForest each tree is built from a separate part of the same training set, reducing the variance, thus contrasting the tendency of a large, single tree to overfit. Given a new instance to classify, the final output is obtained by combining the results given by the different trained models. ACC measures the amount of correctly labelled instances, as classified by a model. It is given by the ratio between the number of correctly classified instances and the number of total instances. WAUC metric is calculated on a ROC curve [61], [62], which is a graphical representation of the sensitivity versus specificity for a classifier system, obtained by varying the model class discrimination threshold. The WAUC value belongs to the interval $[0,1]$; a score of 1 represents the perfect classifier, while 0.5 is typical of a random classification behaviour; this number is computed taking into account also the cardinality/weight of each class. RMSE measures the difference between values predicted by a model and the values actually observed.

By combining the above choices one may end up obtaining as much as 79 feature selection methods, each one of them optimized following a different criterium. In order to choose the best reduced databases, we considered the following multiobjective combinatorial optimization problem:

$$
\begin{array}{ll}
\text { Maximize } & f_{1}(\mathbf{x})=\frac{1}{n} \sum_{j=1}^{n} A C C(\mathbf{x}, j) \\
\text { Maximize } & f_{2}(\mathbf{x})=\frac{1}{n} \sum_{j=1}^{n} W A U C(\mathbf{x}, j) \\
\text { Minimize } & f_{3}(\mathbf{x})=\frac{1}{n} \sum_{j=1}^{n} R M S E(\mathbf{x}, j) \\
\text { Minimize } & f_{4}(\mathbf{x})=\frac{1}{n} \sum_{j=1}^{n} M S(\mathbf{x}, j)
\end{array}
$$

The problem (4) is an instance of the problem (1) with $l=4$ (four objectives) and $m=0$ (no constraints), where $\mathbf{x} \in D B$ is a database $(D B=\{1, \ldots, 79\})$, and $n=3$ is the number of classifiers (J48, RandomForest and LibSVM). The function $A C C(\mathbf{x}, j)$ is the accuracy of the classifier $j$ for the database $\mathbf{x}, W A U C(\mathbf{x}, j)$ is the weighted area under the $R O C$ curve of the classifier $j$ for the database $\mathbf{x}$, $R M S E(\mathbf{x}, j)$ is the root mean squared error of the classifier $j$ for the database $\mathbf{x}$, and $M S(\mathbf{x}, j)$ is the serialized model size of the classifier $j$ for the database $\mathbf{x}$. The solution to the problem (4) is a set of 4 non-dominated databases for INBOUND_AGENTS and a set of 9 non-dominated databases for ALL_AGENTS (all shown in Tab. VII). We compared the performances of these selections (i.e., the best selections obtained with classical methods) against the performances of the selection obtained by the proposed method (hereafter called, generically, MOES-MOEFC-ACC). Tab. VIII shows the result of such a comparison for INBOUND_AGENTS under the accuracy metric (top) and under the area under the $R O C$ metric (bottom) when we tried to learn a classifier with $J 48$, RandomForest, LibSVM, and the multi-objective fuzzy rulebased classifier MOEFC (cfr. Section II), the latter having been run with populationSize set to 100 , generations set to 1000 , maxRules set to 14 , maxLabels set to 7 , maxSimilarity set to $0.1, \min V$ set to 2.0 , and $\max V$ set to 30.0. Tab. IX shows the same comparison ALL_AGENTS. Both comparisons include the respective original dataset (no selection). In both tables, the results of the experiments have been analysed through a paired t-test corrected, with 0.05 significance (being MOES$M O E F C$-ACC the test base). For each result, a mark $*$ denotes that the result is statistically worse than the test base; similarly, a mark $v$ denotes a statistically better result, and no mark denotes no statistically meaningful difference.

Comparing the results with other fuzzy classifiers. Here we compare our classification results with those obtained by other fuzzy rule-based classifiers from the $R$ package frbs [63] and from Weka. The following four algorithms have been considered: FRBCS.CHI [2], FRBCS.W [3], and FH.GBML [4]. FRBCS.CHI extends Wang and Mendel's method [1] for tackling classification problems, and it is quite similar to their technique. However, since it is based on the Fuzzy RuleBased Classification Systems (FRBCS) model, Chi's method only takes class labels on each data to be consequent parts of fuzzy IF-THEN rules. In other words, rules are generated as in Wang and Mendel's technique and, then, the consequents are replaced with their classes. Regarding calculating degrees of each rule, they are determined by the antecedent part of the rules, and redundant rules can be deleted by considering their degrees. FRBCS.W: implements the second type of FRBCS, which has certainty grades (weights) in the consequent parts of the rules. The antecedents are determined by a grid-type fuzzy partition from the training data. The consequent class is defined as the dominant class in the fuzzy subspace corresponding to the antecedents of each fuzzy IFTHEN rule. The class of a new instance is determined by the consequent class of the rule with the maximum product of its compatibility and certainty grades. The compatibility grade is determined by aggregating degrees of the membership function of antecedents, while the certainty grade is calculated from the ratio among the consequent class. FH.GBML is an hybrid algorithm of two fuzzy genetics-based machine learning approaches (i.e., Michigan and Pittsburgh) for designing fuzzy rule-based classification systems. Finally, FURIA [64] (Fuzzy Unordered Rule Induction Algorithm) is implemented as a Weka package that extends the well-known RIPPER rule learner, while preserving its advantages, such as simple and comprehensible rule sets. In addition, it includes a number of modifications and extensions. In particular, FURIA learns fuzzy rules instead of conventional rules and unordered rule 


\begin{tabular}{llll}
\hline$\# I d$ & Database name & Search strategy & Evaluator \\
\hline & & \multicolumn{1}{c}{ INBOUND_AGENTS } \\
\hline$\# 1$ & BF-RF-RMSE & BestFirst & RandomForest $($ RMSE) \\
$\# 2$ & GS-LSVM-ACC & GreedyStepwise & LibSVM (ACC) \\
$\# 3$ & MOES-J48-ACC & MultiObjectiveEvolutionarySearch & J48 (ACC) \\
$\# 4$ & MOES-RF-WAUC & MultiObjectiveEvolutionarySearch & RandomForest (WAUC) \\
\hline \multicolumn{3}{c}{ ALL_AGENTS } \\
\hline$\# 1$ & BF-LSVM-ACC & BestFirst & LibSVM (ACC) \\
$\# 2$ & BF-RF-ACC & BestFirst & RandomForest (ACC) \\
$\# 3$ & BF-RF-RMSE & BestFirst & RandomForest $(R M S E)$ \\
$\# 4$ & LFS-LSVM-WAUC & LinearForwardSelection & LibSVM (WAUC) \\
$\# 5$ & MOES-J48-RMSE & MultiObjectiveEvolutionarySearch & J48 (RMSE) \\
$\# 6$ & MOES-LSVM-RMSE & MultiObjectiveEvolutionarySearch & LibSVM (RMSE) \\
$\# 7$ & MOES-RF-ACC & MultiObjectiveEvolutionarySearch & RandomForest (ACC) \\
$\# 8$ & MOES-RF-RMSE & MultiObjectiveEvolutionarySearch & RandomForest (RMSE) \\
$\# 9$ & PSOS-LSVM-RMSE & PSOSearch & LibSVM (RMSE) \\
\hline & & &
\end{tabular}

TABLE VII

REDUCED DATABASES OBTAINED BOTH PROBLEMS AS A RESULT OF SOLVING (4).

\begin{tabular}{lllllll}
\hline & MOES-MOEFC-ACC & $\mathbf{A 1}$ & $\mathbf{\# 2}$ & $\mathbf{\# 3}$ & $\mathbf{\# 4}$ & INBOUND_AGENTS \\
\hline & \multicolumn{7}{c}{ ACC } \\
\hline MOEFC & 59.40 & $38.53 *$ & $42.67 *$ & 55.23 & 55.17 & $3.33 *$ \\
J48 & 50.10 & 42.80 & 40.13 & 65.60 & 61.57 & 38.90 \\
RandomForest & 45.73 & 42.80 & 53.87 & $67.10 v$ & $68.77 v$ & 56.90 \\
LibSVM & 41.43 & 39.20 & $74.27 v$ & $55.03 v$ & $55.03 v$ & 42.80 \\
ZeroR & 42.80 & 42.80 & 42.80 & 42.80 & 42.80 & 42.80 \\
\hline \multicolumn{7}{c}{ WAUC } \\
\hline MOEFC & 0.63 & $0.50 *$ & 0.53 & 0.60 & 0.59 & $0.50 *$ \\
J48 & 0.62 & $0.50 *$ & 0.55 & 0.74 & 0.72 & 0.58 \\
RandomForest & 0.64 & $0.50 *$ & 0.66 & $0.78 v$ & $0.81 v$ & 0.68 \\
LibSVM & 0.49 & 0.50 & $0.80 v$ & $0.62 v$ & $0.62 v$ & 0.50 \\
ZeroR & 0.50 & 0.50 & 0.50 & 0.50 & 0.50 & 0.50 \\
\hline
\end{tabular}

TABLE VIII

RESULTS OF 10-FOLD CROSS-VALIDATION 10 ITERATIONS FOR THE INBOUND_AGENTS PROBLEM.

\begin{tabular}{|c|c|c|c|c|c|c|c|c|c|c|c|}
\hline & MOES-MOEFC-ACC & $\# 1$ & $\# 2$ & $\# 3$ & $\# 4$ & $\# 5$ & $\# 6$ & \#7 & $\# 8$ & \#9 & ALL_AGENTS \\
\hline \multicolumn{12}{|c|}{$A C C$} \\
\hline$M O E F C$ & 60.12 & $44.89 *$ & 56.77 & 55.91 & $43.98 *$ & $48.21 *$ & 52.07 & 55.00 & 51.62 & $43.64 *$ & $6.25 *$ \\
\hline J48 & 55.00 & 43.55 & 53.75 & 60.87 & 52.43 & $68.43 v$ & 44.79 & 60.27 & 58.21 & 47.18 & 45.98 \\
\hline RandomForest & 59.16 & 54.14 & 66.70 & 64.59 & 52.68 & 57.95 & 54.36 & 69.25 & 68.27 & 54.29 & 56.34 \\
\hline LibSVM & 46.41 & $64.48 v$ & 41.64 & 41.64 & $65.05 v$ & 46.16 & $65.05 v$ & 41.64 & 45.30 & $66.23 v$ & 41.64 \\
\hline ZeroR & 41.64 & 41.64 & 41.64 & 41.64 & 41.64 & 41.64 & 41.64 & 41.64 & 41.64 & 41.64 & 41.64 \\
\hline \multicolumn{12}{|c|}{ WAUC } \\
\hline MOEFC & 0.62 & $0.50 *$ & 0.58 & 0.59 & $0.52 *$ & 0.58 & $0.51 *$ & 0.58 & 0.57 & $0.52 *$ & $0.50 *$ \\
\hline J48 & 0.66 & 0.59 & 0.68 & 0.72 & 0.65 & 0.77 & 0.59 & 0.72 & 0.69 & 0.62 & 0.60 \\
\hline RandomForest & 0.74 & 0.68 & 0.80 & 0.82 & 0.69 & 0.73 & 0.72 & 0.81 & $0.84 v$ & 0.71 & 0.71 \\
\hline LibSVM & 0.54 & $0.71 v$ & 0.50 & 0.50 & $0.72 v$ & 0.56 & $0.72 v$ & 0.50 & 0.53 & $0.73 v$ & 0.50 \\
\hline ZeroR & 0.50 & 0.50 & 0.50 & 0.50 & 0.50 & 0.50 & 0.50 & 0.50 & 0.50 & 0.50 & 0.50 \\
\hline
\end{tabular}

TABLE IX

RESULTS OF 10-FOLD CROSS-VALIDATION 10 ITERATIONS FOR THE ALL_AGENTS PROBLEM.

sets instead of rule lists. Moreover, to deal with uncovered examples, it makes use of an efficient rule stretching method.

The results of comparing multi-objective fuzzy rule-based classification with the models obtained with FRBCS.CHI, FRBCS.W and FH.GBML over the reduced database MOES$M O E F C-A C C$ are shown in Tab. X. We considered the accuracy of the resulting classifiers both in full training and in 10 -fold cross-validation with 10 repetitions, as well as their kappa statistic, number of rules, fuzzy set form (gaussian, trapezoidal, triangular), number of linguistic labels and run time in full training.

Analysis of the results. Tab. VIII and IX can be read in two orthogonal ways:

- First, we may compare how the fuzzy rule-based classifier based on ENORA behaved on selections of features different from MOES-MOEFC-ACC, that is, on the best selections obtained by other methods. In this sense, our selection resulted the most performing one for both problems, beating the second best selection by 7.17 accuracy points in the case of INBOUND_AGENTS and by 3.21 points in the case of ALL_AGENTS. Comparing them by weighted area under the $R O C$ curve shows the same behaviour.

- Second, we may compare the accuracies (and the areas under the $R O C$ curve) of the models learned by different classifiers on the selection MOES-MOEFC-ACC. The fuzzy rule-based classifier obtained, by far, better models than any other classifier, beating the second best one by 


\begin{tabular}{|c|c|c|c|c|c|c|c|c|c|c|}
\hline & MOEFC & FRBCS.CHI & FRBCS.W & FH.GBML & FURIA & MOEFC & FRBCS.CHI & FRBCS.W & FH.GBML & FURIA \\
\hline & \multicolumn{5}{|c|}{ INBOUND_AGENTS } & \multicolumn{5}{|c|}{ ALL_AGENTS } \\
\hline Accuracy full training set & 0.7857 & 0.6607 & 0.7143 & 0.7679 & 0.5357 & 0.7403 & 0.5714 & 0.5714 & 0.6364 & 0.6234 \\
\hline Kappa statistic full training set & 0.666 & 0.485 & 0.5762 & 0.6396 & 0.2385 & 0.5877 & 0.3465 & 0.3465 & 0.4111 & 0.4385 \\
\hline Average accuracy 10-fold CV (10 rep.) & 0.5940 & 0.5544 & 0.5431 & 0.5723 & 0.4649 & 0.6013 & 0.5459 & 0.6004 & 0.5567 & 0.5714 \\
\hline Average kappa statistic 10 -fold CV (10 rep.) & 0.3751 & 0.3018 & 0.296 & 0.3243 & 0.2448 & 0.3749 & 0.2728 & 0.3661 & 0.2761 & 0.3265 \\
\hline Maximum number of rules & 14 & - & - & 14 & - & 14 & - & - & 14 & - \\
\hline Found number of rules & 8 & 21 & 43 & 8 & 3 & 5 & 49 & 46 & 4 & 6 \\
\hline Fuzzy set form & Gaussian & Trapezoidal & Gaussian & Triangular & Trapezoidal & Gaussian & Trapezoidal & Gaussian & Triangular & Trapezoidal \\
\hline Maximum number of linguistic labels for each variable & 7 & 3 & 21 & 14 & - & 7 & 15 & 15 & 4 & - \\
\hline Run time full training set & $36.12 \mathrm{~s}$. & $1.230 \mathrm{~s}$. & $0.399 \mathrm{~s}$. & $4.11 \mathrm{~h}$. & $0.01 \mathrm{~s}$. & $40.5 \mathrm{~s}$. & $1.552 \mathrm{~s}$. & $0.523 \mathrm{~s}$. & $4.86 \mathrm{~h}$. & $0.01 \mathrm{~s}$. \\
\hline Number of generations & 1000 & - & - & 1000 & - & 1000 & - & - & 1000 & - \\
\hline Population size & 100 & - & - & 100 & - & 100 & - & - & 100 & _- \\
\hline Fuzzy sets maximum similarity & 0.1 & _- & _- & - & _- & 0.1 & _- & _- & - & _ \\
\hline
\end{tabular}

TABLE X

COMPARING FUZZY RULE-BASED CLASSIFIERS' METRICS.

9.30 points in the case of INBOUND_AGENTS and by 0.96 points in the case of $A L L_{-} A G E N T S$. It is worth to observe that it is somehow unfair to compare very interpretable classifiers such as the one based on fuzzy rules against non-interpretable ones such as RandomForest; in this sense, in the case of ALL_AGENTS, the difference between the fuzzy model and the next interpretable one, based on a decision tree, is 5.12 points in favour of the former. Moreover, the selection MOES-MOEFC-ACC almost always improves the accuracy of every classifier learning method that we have tried against the original dataset, in both problems.

As far as the behaviour of other fuzzy rule-based classifiers is concerned, the accuracy obtained in full training mode by the multi-objective learner is much better than the one obtained by any other method; such a difference is smaller in cross-validation mode, but it is still there. In terms of interpretability, MOEFC found (much) less rules than $F R$ $B C S . C H I$ and than FRBCS.W, while the number of rules found by FH.GBML is the same (8 rules) in the case of INBOUND_AGENTS, and one less (4 rules instead of 5 rules) in the case of ALL_AGENTS. Although FURIA has found very compact models ( 3 and 6 rules for INBOUND_AGENTS and $A L L \_A G E N T S$ respectively), the accuracy in both full training set and 10-fold cross-validation is much worse than the accuracy obtained with $M O E F C$. Therefore, we can conclude that $M O E F C$ behaves generally better than all three other fuzzy classifiers. In particular, observe that the only classifier whose performances are comparable with those of MOEFC on this problem, that is, FH.GBML, presented a run time several orders of magnitude higher: a few seconds $(M O E F C)$ against a few hours (FH.GBML) with the same number of generations and the same cardinality for the population.

Final model(s). The experiments described and discussed above aimed at comparing the results of the proposed selection method with those of other, classical, selection methods, as well as the performances of the multi-objective fuzzy rulebased learner with those of other, classical, fuzzy rule system learners. In this section we compute a final model over the attributes selected earlier, and we discuss its characteristics in the context of assessing the quality of the agents. For this purpose, the MOEFC classifier has been executed over the $M O E S-M O E F C-A C C$ database with the following parameters:

- We set maxRules to 4 (where 4 is the number of output

\begin{tabular}{lcc}
\hline & INBOUND_AGENTS & ALL_AGENTS \\
\hline Accuracy full training set & 0.7143 & 0.7143 \\
Kappa statistic full training set & 0.5564 & 0.5445 \\
Accuracy 10-fold CV & 0.6607 & 0.6234 \\
Kappa statistic 10-fold CV & 0.4748 & 0.4002 \\
Number of rules & 4 & 4 \\
Fuzzy set form & Gaussian & Gaussian \\
Maximum number of linguistic labels for each variable & 3 & 3 \\
Run time full training set & $1271.59 \mathrm{~s}$. & $1635.38 \mathrm{~s}$. \\
Number of generations & 100000 & 100000 \\
Population size & 100 & 100 \\
Fuzzy sets maximum similarity & 0.4 & 0.4 \\
\hline
\end{tabular}

TABLE XI

PERFORMANCES OF THE FINAL MODELS.

classes) and maxLabels $=3$ (Low, Medium and High), that is, the minimum number of possible rules and a very low number of labels, to maximize the simplicity, that is, the interpretability, of the resulting model;

- We set maxSimilarity to 0.4 , that is, a value sufficiently high to guarantee enough search space, and sufficiently low to guarantee that the linguistic labels do not overlap too much and can be distinguished from each other;

- We set generations to 100000 , to maximize the accuracy.

Tab. XII shows the models, and Tab. XI their performances. For their interpretation, focus, first, on the problem INBOUND_AGENTS. Recall that this is the problem of evaluating the performances (Low, Medium, High or Excellent) of versatile agents, that is, those that manage al types of communications. By interpreting our model, we learn that agents with higher rate of switching among services are generally worse than those with lower rates, but that higher rates of switching among sub-services generically indicates better agents. That is, for agents that deal with all types of communication, switching among services is somehow delicate: changing too often from a service to another negatively influences the (perceived) performances, while changing from a sub-service to another has a positive result. Moreover, and somehow unexpectedly, agents with lower rates of switching and longer turns seem to show better performances.

Let us focus, now, on the problem of classifying agents that manage only outbound communications, that is, specialized agents. The overall daily workload, that is, management, emerges as the most important variable: consistently with the case of INBOUND_AGENTS, the higher workload the better the agent. Moreover, higher rates of icc changes, that is, higher rates of changes, during the day, of the relative importance of the tasks being carried out, as well as too low or too high level of night work, are associated with extreme evaluations 


\begin{tabular}{|c|c|c|c|c|c|c|c|c|c|}
\hline \multicolumn{10}{|c|}{ INBOUND_AGENTS } \\
\hline \multicolumn{10}{|c|}{ Fuzzy rule set } \\
\hline $\mathrm{IF}$ & $x_{1}$ is Moderately High & AND & $x_{2}$ is Moderately Low & AND & $x_{3}$ is Medium & AND & $x_{4}$ is Low & THEN & $y$ is Low \\
\hline IF & $x_{1}$ is Moderately High & AND & $x_{2}$ is Low & AND & $x_{3}$ is Medium & AND & $x_{4}$ is Low & THEN & $y$ is Medium \\
\hline IF & $x_{1}$ is Low & AND & $x_{2}$ is Moderately Low & AND & $x_{3}$ is Medium & AND & $x_{4}$ is High & THEN & $y$ is $H i g h$ \\
\hline IF & $x_{1}$ is Low & AND & $x_{2}$ is Low & AND & $x_{3}$ is $H i g h$ & AND & $x_{4}$ is $H i g h$ & THEN & $y$ is Excellent \\
\hline \multicolumn{10}{|c|}{ Gaussian fuzzy sets } \\
\hline & Attribute & & Name & \multicolumn{2}{|r|}{ Center } & \multicolumn{2}{|r|}{ Standard Deviation } & \multicolumn{2}{|c|}{ Linguistic label } \\
\hline \multirow{2}{*}{\multicolumn{2}{|c|}{$x_{1}$}} & \multirow{2}{*}{\multicolumn{2}{|c|}{ switch_index_ser_type }} & \multirow{2}{*}{\multicolumn{2}{|c|}{$\begin{array}{c}960.7785 \\
3466.7129\end{array}$}} & \multicolumn{2}{|r|}{1152.5188} & \multirow{2}{*}{\multicolumn{2}{|c|}{$\begin{array}{c}\text { Low } \\
\text { Moderately High }\end{array}$}} \\
\hline & & & & & & \multicolumn{2}{|r|}{1286.9339} & & \\
\hline \multirow{2}{*}{\multicolumn{2}{|c|}{$x_{2}$}} & \multicolumn{2}{|c|}{ switch_index_ser_same_type } & \multicolumn{2}{|r|}{0.1893} & \multicolumn{2}{|r|}{$\begin{array}{l}0.6861 \\
0.5784\end{array}$} & \multirow{2}{*}{\multicolumn{2}{|c|}{$\begin{array}{c}\text { Low } \\
\text { Moderately Low }\end{array}$}} \\
\hline & & & & \multicolumn{2}{|r|}{1.5343} & \multicolumn{2}{|r|}{0.5784} & & \\
\hline \multirow{2}{*}{\multicolumn{2}{|c|}{$x_{3}$}} & \multirow{2}{*}{\multicolumn{2}{|c|}{ icc_inbound_av }} & \multicolumn{2}{|r|}{350.6354} & & 203.0594 & \multicolumn{2}{|c|}{ Medium } \\
\hline & & & & \multicolumn{2}{|r|}{798.2903} & & 213.9748 & & High \\
\hline \multirow{2}{*}{\multicolumn{2}{|c|}{$x_{4}$}} & \multirow{2}{*}{\multicolumn{2}{|c|}{ turn_duration }} & & 242.0740 & & 50.3521 & & Low \\
\hline & & & & & 571.1476 & & 84.8794 & & High \\
\hline & & & & $A L L$ & AGENTS & & & & \\
\hline & & & & Fuz & y rule set & & & & \\
\hline$\overline{\mathrm{IF}}$ & $x_{1}$ is Moderately Low & AND & $x_{2}$ is Moderately Low & AND & $x_{3}$ is High & AND & $x_{4}$ is High & THEN & $y$ is Low \\
\hline $\mathrm{IF}$ & $x_{1}$ is Moderately Low & AND & $x_{2}$ is Moderately High & AND & $x_{3}$ is Low & AND & $x_{4}$ is Low & THEN & $y$ is Medium \\
\hline $\mathrm{IF}$ & $x_{1}$ is Moderately High & AND & $x_{2}$ is Moderately Low & AND & $x_{3}$ is Low & AND & $x_{4}$ is Moderately High & THEN & $y$ is High \\
\hline IF & $x_{1}$ is Moderately High & AND & $x_{2}$ is Moderately Low & AND & $x_{3}$ is High & AND & $x_{4}$ is High & THEN & $y$ is Excellent \\
\hline & & & & Gaussi & n fuzzy sets & & & & \\
\hline & Attribute & & Name & & Center & & Standard Deviation & & guistic label \\
\hline & $x_{1}$ & & management & & 257.4638 & & 63.1698 & Mo & derately Low \\
\hline & & & & & 417.3272 & & 91.7403 & $M o$ & derately High \\
\hline & $x_{2}$ & & break_sessions & & 261.3662 & & 164.4175 & Mo & derately Low \\
\hline & & & & & 593.1362 & & 164.4175 & $M o$ & derately High \\
\hline & $x_{3}$ & & icc_inbound_var & & 60345.5613 & & 16629.5431 & & Low \\
\hline & & & & & 42820.7940 & & 34768.2436 & & High \\
\hline & $x_{4}$ & & fraction_night & & 0.0019 & & 0.0017 & & Low \\
\hline & & & & & 0.0053 & & 0.0017 & Mo & derately High \\
\hline & & & & & 0.0086 & & 0.0017 & & High \\
\hline
\end{tabular}

TABLE XII

FUZZY RULE-BASED CLASSIFICATION MODELS.

(Low and Excellent), that is, agents seem to show better performances when they are assigned to both night and day turns, instead of just one.

Guide for parameter settings. Due to the complexity of the proposed method, we finally show a guide for parameter setting in the complete process of feature selection plus fuzzy classification in order to maintain an adequate tradeoff between accuracy and interpretability. The names shown below are the names that appear in the Weka user interface.

\section{1) Feature selection phase:}

a) Search strategy: MultiObjectiveEvolutionarySearch with the following parameters:

- algorithm: ENORA. Although NSGA-II can also be chosen, it has been empirically demonstrated that ENORA obtains better hypervolume values than NSGA-II for feature selection in classification tasks [35], [36].

- generations: 100 (as tested in Section IV Optimal number of generations).

- populationSize: 100 . This population size is widely accepted by the scientific community on Evolutionary Computation.

- reportFrecuency: 100. This parameter establishes the frequency with which the information relative to the population in a generation is printed. It is useful to check the evolution of the algorithm in the testing phase. For the final execution it is convenient to set it equal to the number of generations, thus printing only two reports (at the beginning and at the end).

- seed: 1 . This parameter is necessary for reproducibility. b) Evaluator: WrapperSubsetEval with the following configuration:

- classifier: MultiObjectiveEvolutionaryFuzzyClassifier with the following parameters:

- algorithm: ENORA. Although NSGA-II can also be chosen, it has been empirically demonstrated that ENORA obtains better hypervolume values than NSGAII for classification tasks [25].

- generations: 10 (as tested in Section IV Optimal number of generations).

- populationSize: 100 (as accepted by the scientific community on Evolutionary Computation).

- reportFrecuency: 10 (a report at the beginning and another report at the end).

- evaluationMeasure: ACC. This parameter configures the function $\mathcal{F}_{\mathcal{D}}(\boldsymbol{\Gamma})$ of equation (3) which is used in the optimization process. $A U C$ and RMSE can also be chosen.

- maxLabels: $[3,7]$. This parameter corresponds to $L_{\max }$ in the equation (3). It is assumed that more than 7 linguistic labels lead to a non-interpretable classifier. Set maxLabels $=3$ for maximum compactness.

- maxRules: $[w, \max \{w, 10\}]$. This parameter corresponds to $M_{\max }$ in the equation (3). It is assumed that more than 10 rules lead to a non-interpretable classifier. Set maxRules $=\mathrm{w}$ (number of classes) for maximum compactness. Set maxRules $=\max \{w, 10\}$ for maximum accuracy.

- maxSimilarity: $[0.1,0.4]$. his parameter corresponds to 
$g_{s}$ in the equation (3). It is assumed that a similarity between fuzzy sets greater than 0.4 leads to a noninterpretable classifier. Set maxSimilarity $=0.1$ for a maximum separation between the fuzzy set. Set maxSimilarity $=0.4$ for maximum accuracy.

- $\min V:$ 30.0. This internal parameter sets the value for which the domain of a variable is divided to obtain the minimum variance.

- $\max V:$ 2.0. This internal parameter sets the value for which the domain of a variable is divided to obtain the maximum variance.

- seed: 1 (necessary for reproducibility).

- evaluationMeasure: ACC. This parameter configures the function $\mathcal{F}_{\mathcal{D}}(\mathbf{x})$ of equation (2) which is used in the optimization process. $A U C, R M S E$ (of the class probabilities for discrete class), $M A E$ (mean absolute error of the class probabilities for discrete class), F-measure, and AUPRC (area under the precision-recall curve) can also be chosen.

- folds: 5 (for k-fold cross-validation). A higher number of folds can produce an excessive run time.

- threshold: 0.01. Cross-validation is repeated if standard deviation of mean exceeds this value.

- seed: 1 (necessary for reproducibility).

\section{2) Fuzzy classification phase:}

MultiObjectiveEvolutionaryFuzzyClassifier with the same values of the parameters as in the feature selection phase, with the same parameter values as in the feature selection phase, except the number of generations that must be set to a higher number for a fine tuning of the classifier. Depending on the size of the dataset, we suggest a number of generations between 1000 and 100000 .

\section{CONCLUSIONS AND FUTURE WORKS}

In this work we proposed a novel multivariate feature selection method in which both the search strategy and the classifier are based on multi-objective evolutionary computation. We designed a set of experiments to establish an acceptable setting with respect to the number of evaluations required by the search strategy as well as by the classifier, and we tested our strategy on a real-life dataset. Our proposal is essentially novel: it solves the problem of selecting the best attributes for a very specific classification learning task based on fuzzy rules; as a matter of fact, the performances of a given classifier are very sensible to the attributes that are selected, and using filter selections, which are based on generic statistical values, or wrapper selections obtained with non-fuzzy classifier training not always gives good results. We were able to solve a classification problem in the context of a contact center that required to internally classify the quality of the services being provided, and our classification model turned out to be more accurate and more interpretable than a wide range of non-fuzzy classifier and, also, than other classical fuzzy classifiers.

After the FS phase, all selected attributes are (ideally) used in every rule of a classifier learned by our optimization model. By simply relaxing such a constraint, and by suitably redefining the complexity objective in the optimization model (e.g., by minimizing the sum of the lengths of all rules, or similar measures), the resulting classifiers will encompass rules different subsets of the selected attributes (clearly, the implementation must be adapted to obtain an initial population in which the classifiers have rules of different lengths as well as mutation operators that allow a rule to grow or shrink). It is natural to imagine that such classifiers may be even more accurate, and more interpretable at the same time, and such an improvement is currently considered as possible future work. In addition, we are currently working on the implementation of our own version of multi-objective differential evolution (MODE) for the selection of features and the classification based on rules, as well as their inclusion in the open source software Weka published under the GNU General public license.

\section{ACKNOWLEDGEMENTS}

This study was partially supported by computing facilities of Extremadura Research Centre for Advanced Technologies CETA-CIEMAT), funded by the European Regional Development Fund (ERDF). CETA-CIEMAT belongs to CIEMAT and the Government of Spain. This research was also partially supported by Spanish Ministry of Economy and Competitiveness (Spain) under project TIN2013-45491-R.

\section{REFERENCES}

[1] L. X. Wang and J. M. Mendel, "Generating fuzzy rules by learning from examples," in Proceedings of the 1991 IEEE International Symposium on Intelligent Control, 1991, pp. 263-268.

[2] Z. Chi, H. Yan, and T. Pham, Fuzzy Algorithms: With Applications to Image Processing and Pattern Recognition. River Edge, NJ, USA: World Scientific Publishing Co., Inc., 1996.

[3] H. Ishibuchi and T. Nakashima, "Effect of rule weights in fuzzy rulebased classification systems," IEEE Transactions on Fuzzy Systems, vol. 9, no. 4, pp. 506-515, 2001.

[4] H. Ishibuchi, T. Yamamoto, and T. Nakashima, "Hybridization of fuzzy gbml approaches for pattern classification problems," IEEE Transactions on Systems, Man, and Cybernetics, Part B (Cybernetics), vol. 35, no. 2, pp. 359-365, 2005.

[5] K. Deb, Multi-objective optimization using evolutionary algorithms. London, UK: Wiley, 2001.

[6] M. Gacto, R. Alcalá, and F. Herrera, "Adaptation and application of multi-objective evolutionary algorithms for rule reduction and parameter tuning of fuzzy rule-based systems," Soft Computing, vol. 13, no. 5, pp. 419-436, Dec. 2009.

[7] P. Ducange, B. Lazzerini, and F. Marcelloni, "Multi-objective genetic fuzzy classifiers for imbalanced and cost-sensitive datasets," Soft Computing, vol. 14, no. 7, pp. 713-728, 2010.

[8] P. Ducange, G. Mannara, F. Marcelloni, R. Pecori, and M. Vecchio, "A novel approach for internet traffic classification based on multi-objective evolutionary fuzzy classifiers," in Fuzzy Systems (FUZZ-IEEE), 2017 IEEE International Conference on. IEEE, 07 2017, pp. 1-6.

[9] M. Antonelli, D. Bernardo, H. Hagras, and F. Marcelloni, "Multiobjective evolutionary optimization of type-2 fuzzy rulebased systems for financial data classification," IEEE Trans. Fuzzy Systems, vol. 25, no. 2, pp. 249-264, 2017. [Online]. Available: https://doi.org/10.1109/TFUZZ.2016.2578341

[10] H. Liu and H. Motoda, Feature Selection for Knowledge Discovery and Data Mining. Norwell, MA, USA: Kluwer Academic Publishers, 1998.

[11] E. Amaldi and V. Kann, "On the approximability of minimizing nonzero variables or unsatisfied relations in linear systems," Theoretical Computer Science, vol. 209, no. 1, pp. 237 - 260, 1998. [Online]. Available: http://www.sciencedirect.com/science/article/pii/S0304397597001151

[12] A. G. Karegowda, A. S. Manjunath, and M. A. Jayaram, "Comparative study of attribute selection using gain ratio and correlation based feature selection," International Journal of Information Technology and Knowledge Management, vol. 2, no. 2, pp. 271-277, 2010.

[13] M. A. Hall, "Correlation-based feature selection for machine learning," University of Waikato, Tech. Rep., 1999. 
[14] A. Ahmad and L. Dey, "A feature selection technique for classificatory analysis," Pattern Recognition Letters, vol. 26, no. 1, pp. 43-56, 2005.

[15] R. Kohavi and G. H. John, "Wrappers for feature subset selection," Artificial intelligence, vol. 97, no. 1-2, pp. 273-324, 1997.

[16] N. Japkowicz and M. Shah, Evaluating Learning Algorithms: A Classifcation Perspective. New York, NY, USA: Cambridge University Press, 2011.

[17] C. Wang, M. Shao, Q. He, Y. Qian, and Y. Qi, "Feature subset selection based on fuzzy neighborhood rough sets," Knowledge-Based Systems, vol. 111, pp. 173 - 179, 2016. [Online]. Available: http://www.sciencedirect.com/science/article/pii/S0950705116302714

[18] M. Cintra, H. Camargo, and M.-C. Monard, "Fuzzy feature subset selection using the wang \& mendel method," in Proceedings - 8th International Conference on Hybrid Intelligent Systems, HIS 2008, 10 2008, pp. 590-595.

[19] S. Salzberg, "C4.5: Programs for machine learning by J. Ross Quinlan," Machine Learning, vol. 16, no. 3, pp. 235-240, 1994.

[20] Y. Jin, Ed., Multi-Objective Machine Learning, ser. Studies in Computational Intelligence. Warsaw, Poland: Springer, 2006, vol. 16

[21] J. García-Nieto, E. Alba, L. Jourdan, and E. Talbi, "Sensitivity and specificity based multiobjective approach for feature selection: Application to cancer diagnosis," Information Processing Letters, vol. 109, no. 16 , pp. $887-896,2009$.

[22] J. Zhao, V. B. Fernandes, L. Jiao, I. Yevseyeva, A. Maulana, R. Li, T. Bäck, and M. T. M. Emmerich, "Multiobjective optimization of classifiers by means of 3-d convex hull based evolutionary algorithm," CoRR, vol. abs/1412.5710, 2014

[23] A. Gaspar-Cunha, G. Recio, L. Costa, and C. Estébanez, "Self-adaptive moea feature selection for classification of bankruptcy prediction data," The Scientific World Journal, vol. 2014, 2014.

[24] S. K. Nayak, P. K. Rout, A. K. Jagadev, and T. Swarnkar, "Elitism based multi-objective differential evolution for feature selection: A filter approach with an efficient redundancy measure," Journal of King Saud University - Computer and Information Sciences, 2017.

[25] F. Jiménez, G. Sánchez, and J. M. Juárez, "Multi-objective evolutionary algorithms for fuzzy classification in survival prediction," Artificial Intelligence in Medicine, vol. 60, no. 3, pp. 197-219, 2014.

[26] I. H. Witten, E. Frank, and M. A. Hall, "Introduction to weka," in Data Mining: Practical Machine Learning Tools and Techniques (Third Edition), ser. The Morgan Kaufmann Series in Data Management Systems. Boston: Morgan Kaufmann, 2011, pp. 403 - 406.

[27] T. Hubertus, M. Klaus, and T. Eberhard, Optimization theory. Dordrecht: Kluwer Academic, 2004.

[28] S. Sinha, Mathematical Programming: Theory and Methods. Elsevier Science Limited, 2006.

[29] Y. Collette and P. Siarry, Multiobjective Optimization: Principles and Case Studies. Springer Berlin Heidelberg, 2004.

[30] H. Karloff, Linear Programming. Boston, MA: Birkhauser Basel, 1991.

[31] I. Maros and G. Mitra, Simplex algorithms. Oxford Science, 1996, ch. 1, pp. 1-46.

[32] D. Bertsekas, Nonlinear Programming (Second ed.). Cambridge, MA: Athena Scientific, 1999.

[33] F. Jiménez and J. L. Verdegay, Evolutionary Computation and Mathematical Programming. Heidelberg: Physica-Verlag HD, 2001, pp. 167182.

[34] F. Jiménez, A. Gómez-Skarmeta, G. Sánchez, and K. Deb, "An evolutionary algorithm for constrained multi-objective optimization," in Proceedings of the Evolutionary Computation on 2002. CEC '02. Proceedings of the 2002 Congress, ser. CEC '02, vol. 2. Washington, DC, USA: IEEE Computer Society, 2002, pp. 1133-1138.

[35] F. Jiménez, E. Marzano, G. Sánchez, G. Sciavicco, and N. Vitacolonna, "Attribute selection via multi-objective evolutionary computation applied to multi-skill contact center data classification," in Proc. of the IEEE Symposium on Computational Intelligence in Big Data (IEEE CIBD 15). IEEE, 2015, pp. 488-495.

[36] F. Jiménez, R. Jodár, G. Sánchez, M. Martín, and G. Sciavicco, "Multiobjective evolutionary computation based feature selection applied to behaviour assessment of children," in Proc. of the 2016 International Conference on Educational Data Mining (ICEDM), vol. 2(6), 2016, pp. 1888-1897.

[37] F. Jiménez, G. Sánchez, J. García, G. Sciavicco, and L. Miralles, "Multiobjective evolutionary feature selection for online sales forecasting," Neurocomputing, vol. 234, pp. 75-92, 2017.

[38] K. Deb, A. Pratab, S. Agarwal, and T. Meyarivan, "A fast and elitist multiobjective genetic algorithm: Nsga-ii." IEEE Transactions on Evolutionary Computation, vol. 6, no. 2, pp. 182 - 197, 2002.
[39] F. Jiménez, G. Sánchez, J. García, G. Sciavicco, and L. Miralles, "Multiobjective evolutionary feature selection for online sales forecasting," Neurocomputing, no. 234, pp. 75 - 92, 2017.

[40] V. Kreinovich, C. Quintana, and L. Reznik, "Gaussian membership functions are most adequate in representing uncertainty in measurements," in Proceedings of the NAFIPS\&apos;92. Puerto Vallarta: North American Fuzzy Information Processing Society Conference, 1992, pp. 618-624.

[41] M. Paprzycki, A. Abraham, R. Guo, and S. Mukkamala, "Data mining approach for analyzing call center performance," in Proc. of the 17th International Conference on Industrial and Engineering Applications of Artificial Intelligence and Expert Systems (IEA/AIE), 2004, pp. 10921101.

[42] S. Salcedo-Sanz, M. Naldi, A. Pérez-Bellido, J. Portilla-Figueras, and E. Ortíz-García, "Evolutionary optimization of service times in interactive voice response systems," IEEE Trans. Evolutionary Computation, vol. 14 , no. 4, pp. 602-617, 2010.

[43] E. Zitzler, K. Deb, and L. Thiele, "Comparison of multiobjective evolutionary algorithms: empirical results," Evolutionary Computation, vol. 8, no. 2, pp. 173 - 195, 2000.

[44] J. Novakovic, "Toward optimal feature selection using ranking methods and classification algorithms," Yugoslav Journal of Operations Research, vol. 21, no. 1, 2016.

[45] J. Pearl, Heuristics: Intelligent Search Strategies for Computer Problem Solving. Addison-Wesley Publishing Company, 1984.

[46] S. Russell and P. Norvig, Artificial Intelligence: A Modern Approach, 2nd ed. Prentice-Hall, 2003

[47] M. Gutlein, E. Frank, M. Hall, and A. Karwath, "Large-scale attribute selection using wrappers," in Proceedings of the IEEE Symposium on Computational Intelligence and Data Mining, 2009, pp. 332-339.

[48] S. Dinakaran and P. R. J. Thangaiah, "Role of attribute selection in classification algorithms," International Journal of Scientific \& Engineering Research, vol. 4, no. 6, pp. 67-71, 2013.

[49] A. Moraglio, C. Di Chio, J. Togelius, and R. Poli, "Geometric particle swarm optimization," in Proceedings of the 10th European Conference on Genetic Programming, 2007, pp. 125-136.

[50] D. E. Goldberg, Genetic algorithms in search, optimization and machine learning. Addison-Wesley, 1989.

[51] W. Siedlecki and J. Sklansky, "A note on genetic algorithms for largescale feature selection," Pattern recognition letters, vol. 10, no. 5, pp. $335-347,1989$

[52] H. Vafaie and K. De Jong, "Genetic algorithms as a tool for feature selection in machine learning," in Proceedings of the 4th International Conference on Tools with Artificial Intelligence (TAI), 1992, pp. 200203.

[53] M. A. Hall, "Correlation-based feature selection for machine learning," $\mathrm{Ph} . \mathrm{D}$. dissertation, The University of Waikato, 1999.

[54] H. Liu and R. Setiono, "A probabilistic approach to feature selection a filter solution," in Proceedings of the 13th International Conference on Machine Learning (ICML), vol. 96, 1996, pp. 319-327.

[55] A. G. Karegowda, A. S. Manjunath, and M. A. Jayaram, "Comparative study of attribute selection using gain ratio and correlation based feature selection," International Journal of Information Technology and Knowledge Management, vol. 2, no. 2, pp. 271-277, 2010.

[56] A. Ahmad and L. Dey, "A feature selection technique for classificatory analysis," Pattern Recognition Letters, vol. 26, no. 1, pp. 43-56, 2005.

[57] S. I. Ali and W. Shahzad, "A feature subset selection method based on symmetric uncertainty and ant colony optimization," International Journal of Computer Applications, vol. 60, pp. 5-10, 2012.

[58] R. Schafer, "Accurate and efficient general-purpose boilerplate detection for crawled web corpora," Language Resources and Evaluation, vol. 51, no. 3 , pp. 873-889, 2017

[59] C.-C. Chang and C. J. Lin, "Libsvm: A library for support vector machines," ACM Transactions on Intelligent Systems and Technology, vol. 2, no. 3, 2011

[60] L. Breiman, "Random forests," Machine learning, vol. 45, no. 1, pp. 5-32, 2001.

[61] C. E. Metz, "Basic principles of ROC analysis," in Seminars in nuclear medicine, vol. 8, 1978, pp. 283-298.

[62] T. Fawcett, "An introduction to ROC analysis," Pattern recognition letters, vol. 27, no. 8, pp. 861-874, 2006.

[63] L. Riza, C. Bergmeir, F. Herrera, and J. Benítez, "frbs: Fuzzy rule-based systems for classification and regression in R," Journal of Statistical Software, Articles, vol. 65, no. 6, pp. 1-30, 2015.

[64] J. Hühn and E. Hüllermeier, "Furia: an algorithm for unordered fuzzy rule induction," Data Mining and Knowledge Discovery, vol. 19, no. 3, pp. 293-319, 2009. 